\title{
Funcionalidade familiar de pacientes com Diabetes mellitus no contexto dos serviços de atenção à saúde: protocolo de revisão de escopo
}

\author{
Family functionality of patients with Diabetes mellitus in the context of health care services: scope \\ review protocol
}

Funcionalidad familiar de los pacientes con Diabetes mellitus en el contexto de los servicios de salud: protocolo de revisión del alcance

\begin{abstract}
Resumo
Trata-se de uma revisão de escopo sobre funcionalidade familiar de pacientes com diabetes mellitus adultos no contexto dos serviços de saúde primários, secundários e terciários. O protocolo foi elaborado com base nas orientações do Instituto Joanna Briggs (IJB) e pela metodologia proposta por Arksey e O'Malley além disso, atenderá as recomendações do PreferredReportingItems for Systematicand Meta-Analyses - Extension for ScopingReviews (PRISMA-ScR). Esse protocolo direcionará a sistematização do desenvolvimento da revisão de escopo que terá como objetivo mapear os estudos sobre funcionalidade familiar de pacientes com diabetes mellitus nosserviços de saúde primários, secundários e terciários identificados na literatura nacional e internacional.
\end{abstract}

Palavras-chave: Diabetes Mellitus; Família; Funcionalidade familiar; Serviços de saúde.

\begin{abstract}
This is a scope review on family functionality of patients with adult diabetes mellitus in the context of primary, secondary and tertiary health services. The protocol was prepared based on the guidelines of the Joanna Briggs Institute (IJB) and the methodology proposed by Arksey and O'Malley, in addition, it will meet the recommendations of the PreferredReportingItems for Systematic and Meta-Analyses - Extension for ScopingReviews (PRISMA-ScR). This protocol will direct the systematization of the development of the scope review that will aim to map the studies on family functionality of patients with diabetes mellitus in primary, secondary and tertiary health services identified in the national and international literature.
\end{abstract}

Keywords: Diabetes Mellitus; Family; Family functionality; Health services.

\section{Resumen}

Se trata de una revisión del alcance de la funcionalidad familiar de los pacientes con diabetes mellitus del adulto en el contexto de los servicios de salud primarios, secundarios y terciarios. El protocolo fue elaborado con base en los lineamientos del Joanna Briggs Institute (IJB) y la metodología propuesta por Arksey y O'Malley, además, cumplirá con las recomendaciones del PreferredReportingItems for Systematic and Meta-Analyzes - Extension for ScopingReviews (PRISMA -ScR). Este protocolo orientará la sistematización del desarrollo de la revisión de alcance que tendrá como objetivo mapear los estudios sobre la funcionalidad familiar de los pacientes con diabetes mellitus en los servicios de salud primaria, secundaria y terciaria identificados en la literatura nacional e internacional.

Palabras clave: Diabetes Mellitus; Familia; Funcionalidad familiar; Servicios de salud. 


\section{Introdução}

As doenças crônicas Não Transmissíveis (DCNT) representam um relevante problema de saúde pública, a julgar por serem a principal causa de morte no mundo, ocasionando perdas de vidas prematuras, incapacidades e diminuição da qualidade de vida (Teston, Sales, et al., 2017). As consequências para pacientes, famílias e comunidades são devastadoras, além de sobrecarregar os serviços de saúde. Estudo realizado indica que as populações de baixa renda são mais afetadas por estarem mais vulneráveis e expostas a riscos (Malta et al., 2017)

Neste contexto, situa-se a Diabetes mellitus (DM), que constitui atualmente um dos maiores problemas de saúde mundial. Não apenas pelos altos índices de incidência e prevalência da doença, ou pelos altos custos gerados com serviços de saúde, mas pelas graves complicações e mortes que causa anualmente. As complicações em longo prazo da DM incluem enfermidades cardiovasculares escleróticas, vascular periférica e cerebrovascular, além de nefropatias, constituindo-se em uma das principais causas de deficiências visuais, amputações e insuficiência renal e mortalidade precoce (Mar-García et al., 2017).

A DM é uma desordem que se caracteriza pela elevação dos níveis de açúcar no sangue, chamada de hiperglicemia, que resulta de defeitos na ação ou excreção da insulina no organismo. Pode ser classificada como tipo 1 doença crônica hereditária, que concentra entre 5\% e 10\% do total de diabéticos no Brasil, conhecidos como insulinodependentes; tipo 2 ocorre quando o corpo não aproveita adequadamente a insulina produzida. A causa do diabetes tipo 2 está diretamente relacionado ao histórico familiar, idade superior aos 45 anos, sobrepeso, sedentarismo, triglicerídeos elevados, hipertensão arterial e hábitos alimentares inadequados ((A.D.A.), 2019)

Em comparação com pessoas saudáveis, os pacientes com diabetes, vivenciam uma diminuição na qualidade de vida, devido principalmente ao fato da doença exigir mudanças nos hábitos de vida necessárias para manter um controle metabólico aceitável. Entre elas está o cuidado com a dieta alimentar, a prática de exercícios físicos e o comprometimento com a terapia medicamentosa. Outro fator agravante é a perda da produtividade devido a restrições no tipo de trabalho e quantidade que o indivíduo pode realizar, podendo levar ainda a uma aposentadoria precoce devido as complicações com a doença ocasionando perda de renda pessoal e familiar (Assunção et al., 2017).

Desta forma, à atitude do paciente com relação a doença, deve ser o foco do cuidado, pois os hábitos inadequados de vida e as comorbidades, estão entre os fatores que mais interferem no controle glicêmico. Existe uma tendência de que com o passar do tempo as pessoas com doenças crônicas abandonem as mudanças comportamentais importantes para manter a doença controlada, apresentando um desafio para planejamento da assistência à saúde (Teston, Arruda, et al., 2017)

São evidentes os benefícios do autocuidado adequado dos pacientes com enfermidades crônicas, principalmente o paciente diabético. A DM provoca inúmeras preocupações para à família, sendo que as alterações comportamentais demandam um importante envolvimento e apoio dos familiares. Assim, necessário o acompanhamento da família, nas tomadas de decisões e manejo adequado da doença. Neste contexto, o tratamento pode ser influenciado pela função que à família exerce, seja positiva para auxiliar no tratamento ou negativamente dificultando-o. A família constitui-se em um apoio significativo para a autogestão do cuidado do paciente diabético, mas é crucial que tanto os familiares quanto o paciente estejam devidamente orientados sobre a doença (Mar-García et al., 2017).

Desta forma, são necessárias ações efetivas voltadas para a assistência ao paciente e a sua família, sendo a atenção básica a porta de entrada do sistema de saúde e coordenadora do cuidado (Borges \& Lacerda, 2018).

O objetivo desta revisão de escopo é explorar a literatura existente relacionada à funcionalidade familiar do paciente diabético na atenção primária de saúde, a fim de examinar e mapear conceitualmente as evidências. Identificando desta forma lacunas na literatura sobre o paciente diabético e sua família. A funcionalidade familiar tem ligação inerente com o modo de viver no ambiente familiar dos membros que constituem a família, com o manejo de doenças crônicas não transmissíveis e com a melhor qualidade de vida do paciente (Vera, 2021). A metodologia do Joanna Briggs Institute (JBI) será usada para a 
realização desta revisão de escopo (Peters et al., 2020). Foi realizada uma pesquisa preliminar de PROSPERo, Cochrane Database of Systematic Reviews e Joanna Briggs Institute (JBI) Evidence Synthesis. Não foram identificadas revisões de escopo ou revisões sistemáticas existentes ou em andamento sobre esse tópico.

\subsection{Pergunta de pesquisa}

Que conceitos existem na literatura sobre a funcionalidade familiar de pacientes com Diabetes mellitus, no contexto dos serviços de saúde primários, secundários e terciários?

- Descrever os conceitos encontrados na literatura sobre a funcionalidade familiar dos pacientes com Diabetes mellitus nos serviços de saúde primários, secundários e terciários.

\subsection{Critérios de inclusão}

\subsubsection{Participantes}

Esta revisão de escopo considerará estudos que incluem pacientes adultos com idade entre 20 e 59 anos, com Diabetes mellitus e sua família, em todo o mundo, que sejam atendidos no contexto dos serviços de saúde primários, secundários e terciários.

\subsubsection{Conceito}

O conceito de interesse é a funcionalidade familiar. Família pode ser definida como aqueles que dizem que são ou que formam uma família (Friedman \& Paavilainen, 2003; Wriigth \& Leahey, 2012). Duas ou mais pessoas que são ligadas por íntimas associações, recursos e valores e é reconhecida quando os membros consideram os elementos que a constituem como uma família (Perri J. Bomar, 2004). O funcionamento familiar refere-se ao conjunto de relações interpessoais que ocorrem em cada família, o que lhes proporciona identidade própria (Friedmann, 2003). Funcionalidade familiar é retratada em formas de companheirismo, afetividade, ajustamento e capacidade de resolução da família junto aos seus membros(Sousa-Muñoz \& Sá, 2020).

\subsubsection{Contexto}

Esta revisão de escopo avaliará estudos conduzidos no ambiente dos serviços de saúde primários, secundários e terciários, em regiões urbanas e rurais (contexto global), bem como o ambiente familiar do paciente com diabetes mellitus. O foco está na atenção primária à saúde e contexto familiar para adultos. No contexto primário encontram-se as Unidades Básicas de Saúde (UBS). O contexto secundário é composto pelas Unidades de Pronto Atendimento (UPA), os ambulatórios de especialidades e o contexto terciário é composto pelos hospitais.

\subsubsection{Tipos de fonte}

Esta revisão de escopo considerará desenhos de estudos experimentais, incluindo estudos controlados randomizados, estudos controlados não randomizados. Além disso, estudos observacionais analíticos, incluindo estudos de coorte prospectivos e retrospectivos, estudos de caso-controle e estudos transversais analíticos serão considerados. Esta revisão também irá considerar desenhos de estudos observacionais descritivos, incluindo séries de casos, relatos de casos individuais e estudos transversais descritivos para inclusão. Serão considerados desenhos de estudos qualitativos, incluindo, mas não se limitando a, fenomenológica, teoria fundamentada, etnográfica, descrição qualitativa, pesquisa-ação e pesquisa feminista. Além disso, revisões sistemáticas, dissertações e literatura cinzenta, bem como textos e artigos de opinião, serão 
considerados para inclusão. Pesquisa que inclui uma análise de subgrupo, relacionada ao contexto, conceito ou população, também será considerada para inclusão.

\section{Metodologia}

A revisão do escopo proposta será conduzida de acordo com o método da Joanna Briggs Institute (JBI) (Peters et al., 2020).

\subsection{Estratégia de pesquisa}

A estratégia de busca terá como objetivo localizar estudos publicados e não publicados. A estratégia de busca será realizada em três etapas nesta revisão. Será realizada uma busca inicial limitada no MEDLINE (PubMed) e CINAHL (EBSCO) para identificar os artigos, seguida da análise das palavras do texto incluídas no título e no resumo e dos termos do índice usados para descrever o artigo. Logo será realizada uma segunda pesquisa usando todas as palavras-chave e termos de índice identificados em todos os bancos de dados incluídos. Na terceira fase será realizada uma busca nas listas de referência de todos os relatórios e artigos incluídos após a triagem de texto completo serão examinadas para identificar estudos adicionais. Uma estratégia de busca proposta para Pub Med é detalhada a seguir:

$1^{\circ}$ passo conjunto $\mathrm{P}$ :

(Pacient* OR usuári* OR indivídu* OR pesso* OR doent* OR client* OR Patient OR user OR individual OR person OR patient OR client OR Paciente OR usuario OR individuo OR persona OR paciente OR cliente OR insulin*) AND (diabet* OR "diabetes mellitus" OR "diabetes mellitus tipo 2" OR "diabetes mellitus tipo 1" OR "complicações do diabetes" OR "diabetes mellitos dependente de insulina" OR "diabetes mellitus dependente" OR "diabetes mellitus dependente de insulina” OR "diabetes mellitus não dependente tipo 4" OR "Síndrome de Donohue” OR Síndrome de Wolfram” OR OR "diabetes mellitus type 2" OR "diabetes mellitus type 1" OR "complicationsof diabetes" OR "diabetes mellitus insulindependent" OR "diabetes mellitus dependent" OR "diabetes mellitus insulindependent" OR "Type 4 non-dependent diabetes mellitus" OR "Donohue'sSyndrome" OR Wolfram'sSyndrome" OR diabetes OR "complicaciones de la diabetes" OR "diabetes mellitus dependiente de insulina" OR "diabetes mellitus dependiente" OR "diabetes mellitus dependiente de insulina" OR "Tipo 4 diabetes mellitus no dependiente)

\section{$2^{\circ}$ passo conjunto I:AND}

(famil* OR " ciclo de vida familiar" OR " ciclo de vida, família" OR "membros da família" OR "membro da família" OR “ famílias adotivas" OR "família reconstituída” OR “ famílias reconstituídas” OR filiaçã* OR "redes de parentesco" OR "rede de parentesco" OR parent* OR "família extendida" OR "famílias extendidas" OR “ pesquisa familiar" OR "pesquisa, família" OR familia OR "ciclo de vida familiar" OR "ciclo de vida, familia" OR "miembros de lafamilia" OR "miembro de lafamilia" OR "familias de acogida" OR "familiareconstituida" OR "familiasreconstituidas" OR afiliación OR "redes de parentesco" OR "red de parentesco" OR padre OR "familia extensa" OR "familiasextendidas" OR "búsqueda de familia" OR "búsqueda, familia" OR family* OR "familylifecycle" OR "lifecycle, family" OR "familymembers" OR "familymember" OR "fosterfamilies" OR "reconstitutedfamily" OR "reconstitutedfamilies" OR affiliation OR " kinship networks" OR "kinship network" OR parent* OR "extendedfamily" OR "extendedfamilies" OR "familysearch" OR "search, family") (funciona* OR autoeficácia OR relaçã* OR exito OR efetividade OR ação OR efeito OR eficiência OR eficácia OR valência OR valia OR prestim* OR benefici* OR efeit* OR competênci* OR works OR self-efficacy OR relation OR success OR effectiveness OR action OR effect OR efficiency OR effectiveness OR valence OR value OR helpfulness OR beneficial OR 
effect OR competence OR unciona OR autoeficacia OR relación OR éxito OR efectividad OR acción OR efecto OR eficiencia OR efectividad OR valencia OR valor OR utilidad OR beneficioso OR efecto OR competencia) AND

$3^{\circ}$ passo - conjunto c

(“atenção primária à saúde" OR "atenção primária" OR "atenção básica” OR "atenção básica à saúde” OR "cuidados primários" OR "cuidados de saúde primários" OR "cuidados secundários" OR “ centros de cuidados secundários" OR ambulatóri* OR "pacientes ambulatórias" OR "ambulatório hospitalar" OR "ambulatórios hospitalares" OR "cuidado ambulatório" OR "cuidado ambulatorial” OR " monitoramento ambulatorial” OR cuidados de saúde terciários" OR "hospital" OR "hospitais" OR "Home Care" OR "cuidados paliativos" OR "cuidado terminal” OR “ cuidados de longo prazo" OR "primaryhealthcare" OR "primarycare" OR "basiccare" OR "basichealthcare" OR "primarycare" OR "primaryhealthcare" OR "secondarycare" OR "secondarycare centers" OR outpatients OR "hospital outpatient" OR "hospital outpatient" OR "outpatientcare" OR "outpatientcare" OR "outpatientmonitoring" OR "tertiaryhealthcare" OR "hospital" OR "hospital" OR "Home Care" OR "carepalliatives" OR "terminal care" OR "long-termcare" OR “atención primaria de salud" OR "atención primaria” OR "atención básica" OR "atención primaria de salud" OR "atención primaria” OR "atención primaria de salud” OR “atención secundaria” OR “centros de atención secundaria” OR "pacientes ambulatórios" OR "pacientes ambulatórios" OR "hospital ambulatorio" OR atenciónambulatoria" OR "monitoreo ambulatório" OR "atención terciaria de salud" OR “hospital” OR “atención domiciliaria” OR "cuidados paliativos” OR “terminal atención” OR “atención a largo plazo

A estratégia de busca, incluindo todas as palavras-chave e termos de índice identificado, será adaptada para cada fonte de informação incluída. Os termos de pesquisa relacionados à população de interesse incluem "diabetes mellitus", "família", "Atenção primária a saúde ". Outras palavras-chave incluem "unidade familiar" e "atenção básica". Será considerada a literatura publicada em todos os idiomas, e os bancos de dados serão pesquisados desde o início das publicações até o mês de dezembro de 2021.

As fontes de banco de dados de informações incluem PubMed / MEDLINE (Ciências da Saúde); CINAHL (EBSCO), MEDLINE (Ovid);BDENF (Enfermagem; américa latina); COCHRANE Library (Ciências da Saúde / Medicina baseada em evidências); Embase (Ciências da Saúde); LILACS / BVS (Literatura Latino-Americana e do Caribe em Ciências da Saúde); SCiELO (multidisciplinar; inclui principalmente revistas latino-americanas); Teses e dissertações (ProQuestDissertations\&Theses/ Networked Digital Library ofThesesandDissertations/ Biblioteca Digital Brasileira de Teses e Dissertações - BDTD); Scopus (multidisciplinar; abrangência mundial); Web of Science (multidisciplinar; abrangência mundial).

\subsection{Seleção de estudos}

Após a pesquisa, todas as citações identificadas serão agrupadas e carregadas no gerenciador de referências End Note onde as duplicatas serão removidas. Primeiramente a seleção será realizada com base nos títulos e resumos, por dois revisores independentes de acordo com $o s$ critérios de inclusão para a revisão. Os estudos serão selecionados no software RYYAN QCRI, que permite o trabalho simultâneo cegado dos dois revisores e a intervenção de um terceiro revisor que será o supervisor da revisão e responsável pelo loguin do software. Estudos potencialmente relevantes serão recuperados na íntegra e seu detalhe de citação importado para o gerenciador End note. O texto completo das citações selecionadas será avaliado em detalhes em relação aos critérios de inclusão por dois revisores independentes. Serão registrados motivos para a exclusão de estudos de texto completo que não atendam aos critérios de inclusão. Quaisquer divergências que surjam entre os revisores em cada etapa do processo de seleção do estudo serão resolvidas por meio de discussão ou com o terceiro revisor. Os resultados da pesquisa 
serão relatados na íntegra e apresentados em um diagrama de fluxo de Itens de Relatório Preferidos para Revisões Sistemáticas e Meta-análises para revisões de escopo (PRISMA-ScR).

\subsection{Extração de dados}

Os dados serão extraídos dos artigos selecionados por dois revisores independentes usando a ferramenta de extração de dados desenvolvida pela JBI, que foi adaptada para uma revisão de escopo pelos revisores. Uma ferramenta preliminar de extração de dados habitual foi incluída no Apêndice II. A ferramenta será testada e comparada por dois revisores concluindo extrações independentemente, caso se mostre apta, seguirá o processo de extração. Os dados a serem extraídos incluirão informações sobre o artigo, objetivo do artigo, população, conceito, contexto do estudo, métodos do estudo e principais resultados relevantes para o objetivo da revisão. Uma descrição detalhada do processo de extração de dados, a ferramenta de extração de dados e as modificações da ferramenta serão incluídas no relatório de revisão de escopo completo. As divergências que ocorrem entre os revisores serão mediadas por meio de discussão ou com um terceiro revisor. Os autores das fontes serão contatados para solicitar dados em falta ou adicionais, caso seja necessário.

\subsection{Análise e apresentação de dados}

Os dados analisados serão apresentados em forma de diagrama alinhado com o objetivo desta revisão de escopo. Um resumo narrativo acompanhará os resultados tabulados ou mapeados para descrever as características da literatura e como os resultados se inter-relacionam com o objetivo e a questão de pesquisa. As categorias usadas para apresentação de dados incluem aquelas dentro da ferramenta de extração; no entanto, eles podem ser modificados com base nas conclusões da revisão.

\section{Registro da revisão de escopo: $\underline{\text { osf.io/245px }}$}

\section{Agradecimentos}

A Dda. Marinéia Kickhofel pela colaboração na leitura e revisão do protocolo de revisão.

\section{Referências}

(A.D.A.), A. D. (A. D. A. (2019). Diabetes Care. Journal of Clinical and Aplied Research and Education, 42(1). http://library1.nida.ac.th/ter mpaper6/sd/2554/19755.pdf

Assunção, S. C., Fonseca, A. P., Silveira, M. F., Caldeira, A. P., \& Pinho, L. de. (2017). Knowledge and attitude of patients with diabetes mellitus in Primary Health Care. Escola Anna Nery, 21(4), 1-7. https://doi.org/10.1590/2177-9465-ean-2017-0208

Borges, D. de B., \& Lacerda, J. T. de. (2018). Ações voltadas ao controle do Diabetes Mellitus na Atenção Básica: proposta de modelo avaliativo. Saúde Em Debate, 42(116), 162-178. https://doi.org/10.1590/0103-1104201811613

Friedemann, M. L., \& Paavilainen, E. (2003). Development of a Family Assessment Instrument for Transcultural Use. Journal of Transcultural Nursing, 14(2), 90-99. https://doi.org/10.1177/1043659602250612

Malta, D. C., Bernal, R. T. I., Lima, M. G., Araújo, S. S. C. de, Silva, M. M. A. da, Freitas, M. I. de F., \& Barros, M. B. de A. (2017). Doenças crônicas não transmissíveis e a utilização de serviços de saúde: análise da Pesquisa Nacional de Saúde no Brasil. Rev Saude Publica, 51(1), 1-10. https://doi.org/10.1590/S1518-8787.2017051000090

Mar-García, J., Peñarrieta-de Córdova, I., León-Hernández, R., Gutiérrez-Gómez, T., Banda-González, O., Rangel-Torres, S., \& de León-Ramírez, M. (2017). Relación entre automanejo y percepción de funcionalidad familiar en personas con diabetes mellitus tipo 2. Enfermería Universitaria, 14(3), 155-161. https://doi.org/10.1016/j.reu.2017.05.005

Perri J. Bomar. (2004). Promoting Health in Families: Applying Family Research and Theory to Nursing (3rd ed.). W.B. Saunders Company.

Teston, E. F., Arruda, G. de O. de, Sales, C. A., Serafim, D., \& Marcon, S. S. (2017). Consulta de enfermagem e controle cardiometabólico de diabéticos: ensaio clínico randomizado. Rev Bras Enferm, 70(3), 492-498. http://dx.doi.org/10.1590/0034-7167-2016-0352 
Research, Society and Development, v. 10, n. 13, e96101320332, 2021

(CC BY 4.0) | ISSN 2525-3409 | DOI: http://dx.doi.org/10.33448/rsd-v10i13.20332

Sousa-Munoz, R. L. de, \& Sá, A. D. de. (2020). Apoio social, funcionalidade familiar e controle glicêmico de pacientes diabéticos tipo 2. Revista De Medicina, 99(5), 432-441. https://doi.org/10.11606/issn.1679-9836.v99i5p432-441

Teston, E. F., Sales, C. A., \& Marcon, S. S. (2017). Perspectives of individuals with diabetes on selfcare: contributions for assistance. Escola Anna Nery Revista de Enfermagem, 21(2), 1-8. https://doi.org/10.5935/1414-8145.20170043

Vera, I. (2021). Relações familiares de idosos: estudo populacional Family relationships of the elderly: a population-based study. 44376-44389. https://doi.org/10.34117/bjdv7n5-047

Wright, L., \& Leahey, M. (2012). Enfermeiras e famílias: guia para avaliação e intervenção na família (5th ed.). ROCA. 